\title{
INFLUÊNCIA DA VARIABILIDADE CLIMÁTICA GLOBAL E DE SUAS ESCALAS TEMPORAIS SOBRE A PRECIPITAÇÃO NO ALTO MUNDAÚ (PE)
}

\author{
Djane Fonseca da Silva ${ }^{1,}$ Francisco de Assis Salviano de Sousa ${ }^{2}$, Mary Toshie \\ Kayano $^{3}$, Josiclêda Domiciano Galvíncio ${ }^{4}$
}

Artigo recebido em $23 / 10 / 2009$ e aceito em 07/01/2010

\section{RESUMO}

As variações da precipitação em uma região da bacia hidrográfica do rio Mundaú foram estudadas utilizando o Índice de anomalia de chuva (IAC) e a Análise de Ondeleta. Esta técnica envolve transformação de uma série unidimensional em um espaço de tempo e freqüência, permitindo determinar as escalas de variabilidade dominantes e suas variações temporais. Os resultados mostraram que a variabilidade da precipitação é definida por multi-escalas temporais localizadas em certos intervalos de tempo. No entanto, a variabilidade interanual ligada ao ciclo de El Niño Oscilação Sul e a variabilidade decadal influenciaram na variabilidade pluviométrica do Alto Mundaú.

Palavras-chave: Variabilidade Decadal, Ponto de Inversão, Escalas Temporais, IAC, Análise de Ondeletas.

\section{EFFECTS OF GLOBAL CLIMATE VARIABILITY AND THEIR TIME ON THE SCALE PRECIPITATION IN THE UPPER MUNDAÚ (PE)}

\begin{abstract}
The variations of the precipitation in a region of the hydrographic basin of the river Mundaú had been studied using the RAI and the Wavelet Analysis. This technique involves transformation of a one-dimensional series in a time space and frequency, allowing to determine the dominant scales of variability and its secular variations. The results had shown that the precipitation variability is defined by located secular multiscales in certain intervals of time. However, on interanual variability to the ENSO cycle and the decadal variability had influenced in the pluviometric variability of the "Upper" Mundaú.

Palavras-chave: Variabilidade Decadal, Ponto de Inversão, Escalas Temporais, IAC, Análise de Ondeletas.

\footnotetext{
${ }^{1}$ Pós-Doutoranda no Curso de Geografia (CFCH)-UFPE, Av. Prof. Moraes Rego, 1235 - Cidade Universitária, Recife (PE)-CEP: 50670-901, e-mail: djanefonseca@ yahoo.com.br

${ }^{2}$ Prof. Dr. Departamento de Ciências Atmosféricas-UACA/ UFCG, e-mail: fassis@dca.ufcg.br

${ }^{3}$ Prof. Dr. e pesquisadora CPTEC/INPE (SP), e-mail: mary.kayano@cptec.inpe.br;

${ }^{4}$ Prof. Dr. Curso de Geografia (CFCH)-UFPE, e-mail: josicleda@ hotmail.com
} 


\section{INTRODUÇÃO}

O rio Mundaú, o principal da bacia, nasce a oeste da cidade pernambucana de Garanhuns, na parte sul do Planalto da Borborema e entra em Alagoas na Cachoeira da Escada, situada ao sul da cidade de Correntes e noroeste da cidade alagoana de Santana do Mundaú. Depois, o rio Mundaú atravessa a área central da Mata Alagoana e chega ao litoral, com a sua foz afogada formando a Lagoa Mundaú (TENÓRIO, 1985).

Os meses de fevereiro a julho correspondem à aproximadamente $73 \%$ de toda precipitação anual local. $\mathrm{O}$ período chuvoso da região coincide com a época em que há atuação de Ondas de Leste, que somadas com sistemas de escala local (Convergência dos Alísios e Brisas), intensificam as chuvas, principalmente à noite (COSTA et al., 2005).

Tal bacia hidrográfica situa-se na região nordeste do Brasil (NEB) que é conhecida por apresentar em alguns anos secas severas ou chuvas excessivas que, por sua vez, têm sido relacionadas aos padrões anômalos de grande escala da circulação atmosférica global associados ao fenômeno El-NiñoOscilação Sul (ENOS).
As secas severas no NEB, objeto de inúmeros estudos por seus impactos sociais e econômicos, têm sido relacionadas à ocorrência do El Niño. Esta hipótese é baseada no fato de que para alguns anos de El Niño (fortes ou moderados) ocorrem secas severas no NEB (ANDREOLI et al., 2004). Entretanto, a relação entre El Niño e as secas no NEB não é unívoca (KANE e TRIVEDI, 1988; KANE, 1992; KANE, 1997). Kane (1997) mostrou que dos 46 El Niño (fortes e moderados) do período de 1849-1992, somente 21 (45\%) estiveram associados a secas severas em Fortaleza, por exemplo.

Apesar da variabilidade de TSM no Pacífico ser dominada por variabilidades na escala de tempo interanual, Zhang et al. (1997) separaram a variabilidade temporal do Pacífico em duas componentes: uma relacionada à escala de variabilidade interanual do ciclo ENOS e a outra, linearmente independente incluindo toda a variabilidade interdecadal.

Haja vista que as análises espectrais das séries de anomalias de temperatura de superfície do mar (ATSM) para os Oceanos Atlântico e Pacífico são caracterizadas por oscilações em escalas de tempo 
interanual e decadal, sendo a escala interanual mais pronunciada na região do Pacífico e a decadal, no Atlântico (HASTENRATH E KACZMARCZYK, 1981; SPERBER e HAMEED, 1993; MEHTA e DELWORTH, 1995), pretende-se neste trabalho verificar quais escalas exercem influência sobre a variabilidade pluviométrica da região do Alto Mundaú (AM), cabeceiras do rio Mundaú, no estado de Pernambuco, situada na região nordeste do Brasil.

Assim, as variações da precipitação na região de estudo serão estudadas utilizando a Análise de Ondeleta (AO). Esta técnica envolve transformação de uma série unidimensional em um espaço de tempo e frequiência, o que permite determinar as escalas de variabilidade dominantes $\mathrm{e}$ suas variações temporais.

Sabe-se que a precipitação pluviométrica no NEB é resultante do acoplamento de diversos sistemas atmosféricos de várias escalas quase periódicos que podem ser modificados pelas características fisiográficas da região e por anomalias atmosféricas de escala planetária. Destacam-se o dipolo do Atlântico e o ENOS, que modificam freqüência, distribuição espacial e intensidade desses sistemas; deste modo, faz-se necessário estudar a variabilidade interanual, com ênfase na quadra chuvosa de ambas as regiões.

\section{2 Índice de Anomalia de chuva e Análise de Ondeletas}

No NEB a previsão e o monitoramento de períodos de secas ou chuvosos são particularmente úteis devido aos seguintes aspectos: (1) a existência de inúmeros projetos de irrigação implantados e a serem implantados ao longo dos principais rios; (2) o abastecimento de água das grandes cidades é, em sua maioria, dependente direto do escoamento dos rios, ou indiretamente do volume acumulado nas barragens; (3) a maioria das culturas agrícolas depende exclusivamente da regularidade das chuvas e (4) a possibilidade de uso de água subterrânea é pequena quando comparada ao da água superficial (FREITAS, 2004; 2005).

Deste modo, o monitoramento desses períodos pode ser efetuado através do emprego de índices. Com base neles, pode-se desenvolver um sistema de acompanhamento das características dos períodos de seca ou chuvosos, assim como as diferenciadas medidas a serem efetivadas de acordo 
com os valores atingidos por tais parâmetros.

Freitas (2004; 2005) utilizou o IAC para localidades no estado do Ceará e observou que é possível fazer uma comparação das condições atuais de precipitação em relação aos valores históricos, servindo ainda para avaliar a distribuição espacial do evento, consoante sua intensidade.

Mauget (2005) ao estudar a variação multi-decadal da precipitação de 1901 a 1998 para identificar as concentrações mais significativas de anos úmidos e secos dentro da série, em regiões continentais, encontrou alta incidência de anos úmidos na América do Norte durante 1972 a 1998, com oito dos dez anos mais úmidos desde 1901, ou seja, oito eventos aconteceram durante esse último período de 27 anos.

Gonçalves et al. (2006) obtiveram o IAC para 15 estações situadas no rio São Francisco, fato que mostrou que a incidência de anos secos é substancialmente maior na região estudada. Através da aplicação do índice em áreas situadas à jusante da hidrelétrica de Sobradinho, pode-se explicar a ocorrência de cheias e inundações ocorridas no sertão pernambucano.
Segundo Repelli et al. (1998), o índice proposto parece ser apropriado para utilização em regiões semi-áridas e ou tropicais, especialmente para o NEB. A facilidade de acesso aos dados de precipitação mensais em tempo real é um fator importante e faz do índice uma potencial ferramenta para aplicações de monitoramento durante a estação chuvosa.

Estudos que utilizam as ondeletas vêm crescendo em várias áreas. Desde a década de 1990 que a aplicação da Transformada em Ondeletas (TO) vem sendo utilizada em diversas áreas da ciência e da técnica, desde as ciências médicas às ciências exatas, da eletrônica à ótica aplicada (Vitorino, 2003). Assim, para se analisar variações decadais nas séries, pode-se utilizar a TO. A partir destas pode-se detectar oscilações de 12 anos como de 3-7 anos (Tucci e Braga, 2003) e gerar uma medida quantitativa (frequiência) de mudanças ao longo do tempo e determinar o período total dessas mudanças.

Segundo Barbosa (2004), basicamente, a idéia central da análise de ondeletas (AO), no contexto de análises de sinais, consiste em decompor uma série temporal em 
diferentes níveis de resolução tempofreqüência e então determinar as componentes da variabilidade dominantes.

$\mathrm{Na}$ meteorologia, houve grande quantidade de trabalhos aplicando a $\mathrm{AO}$ ao sinal turbulento (FARGE, 1992). No que se refere à climatologia e à dinâmica atmosférica, poucos estudos utilizaram até o momento a AO. A grande maioria dos trabalhos desenvolvidos na dinâmica atmosférica enfoca as escalas climáticas, desde a sua variabilidade interdecadal a intrasazonal. Em geral, estes trabalhos buscam uma metodologia eficaz na localização de fenômenos transientes e que atuem simultaneamente em várias escalas de tempo, proporcionando novas perspectivas e abordagens de análises, impossíveis de se efetuarem por meio de técnicas tradicionais.

A AO é útil para detectar, analisar e caracterizar as escalas de tempo que afetam os sistemas atmosféricos sobre a América do Sul e oceanos adjacentes. Esta ferramenta revela a estrutura temporal das séries temporais nãoestacionárias. O reconhecimento que a AO possui propriedades matemáticas capazes de quantificar as relações tempo $\mathrm{X}$ escala em meteorologia, estimula muitas pesquisas em mesoescala e na escala sinótica (REPELLI et al., 1998; SCHNEIDER et al., 2005).

Alguns autores associam eventos na escala interdecadal ao ciclo de manchas solares. Eles relataram muitos fenômenos geofísicos que também apresentam periodicidades desta ordem e tentaram estabelecer uma relação destes com o ciclo solar de 10-11 anos. Todavia, tal explicação foi duramente contestada por autores, tais como Mann et al. (1995), para os quais tal variabilidade climática, pelo menos no Hemisfério Norte, deva ser atribuída à interação entre o Oceano Atlântico e a atmosfera, ponto de vista que é parcialmente endossado por Allen e Smith (1994). Todavia, Kerr (1996) discute novas evidências a partir das quais a conexão sol-clima deve ser considerada seriamente, particularmente no que se refere às flutuações de temperatura dos oceanos tropicais e subtropicais.

Andreoli et al. (2004) utilizaram a TO para analisar as variações da precipitação em Fortaleza e da TSM nos oceanos Pacífico e Atlântico para o período de 1856 a 1991 e foi identificado um pico dominante de 12,7 anos na série. Confirmou-se, ainda, alta 
coerência entre a variabilidade de precipitação no norte do NEB e o gradiente inter-hemisfério de ATSM no Atlântico na escala decadal.

\section{MATERIAL E MÉTODOS}

A bacia hidrográfica do rio Mundaú tem uma área total de 4.126 $\mathrm{km}^{2}$, está compreendida entre as latitudes de $9,4^{\circ}$ a $8,4^{\circ} \mathrm{S}$ e longitudes $35,4^{\circ}$ e $36,2^{\circ} \mathrm{W}$, abrangendo os Estados de Alagoas e Pernambuco, conforme a Figura 1.

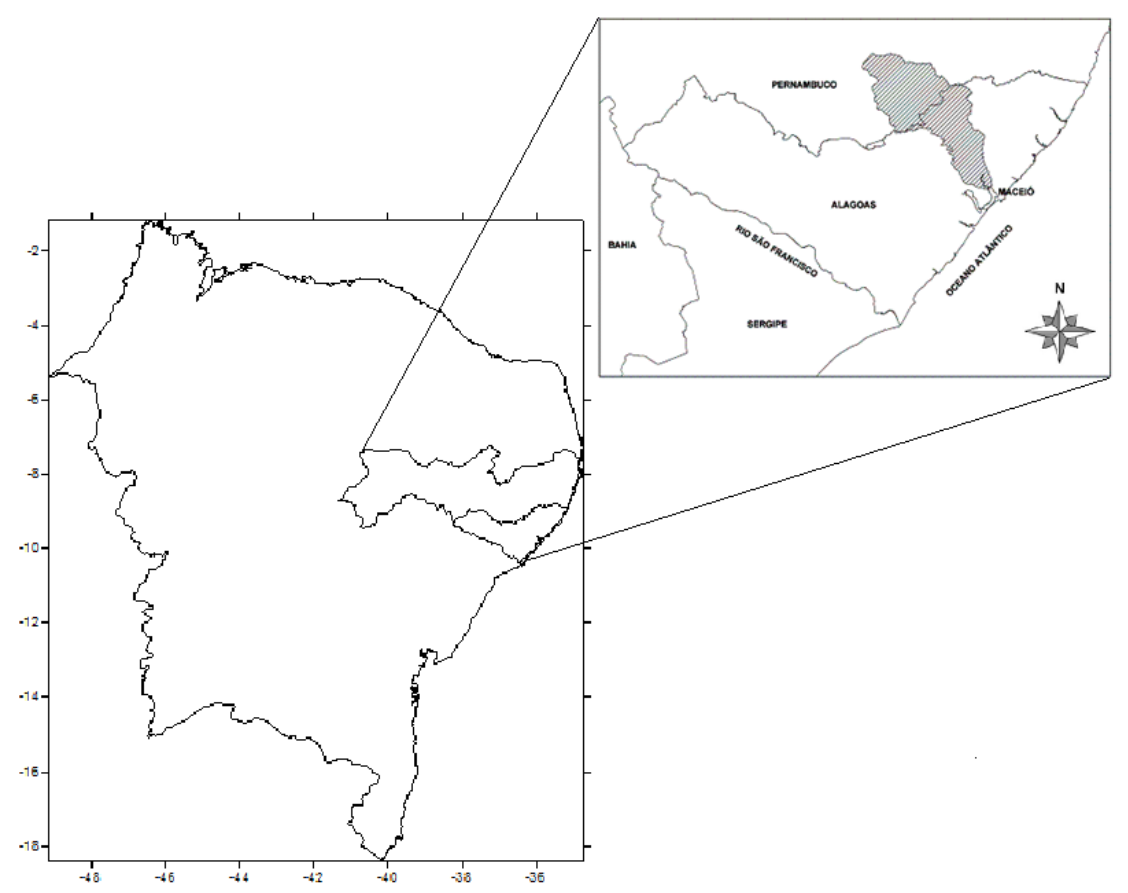

Figura 1: Localização da bacia hidrográfica do rio Mundaú em relação aos Estados que a compõem, Alagoas e Pernambuco, e em relação ao nordeste brasileiro.

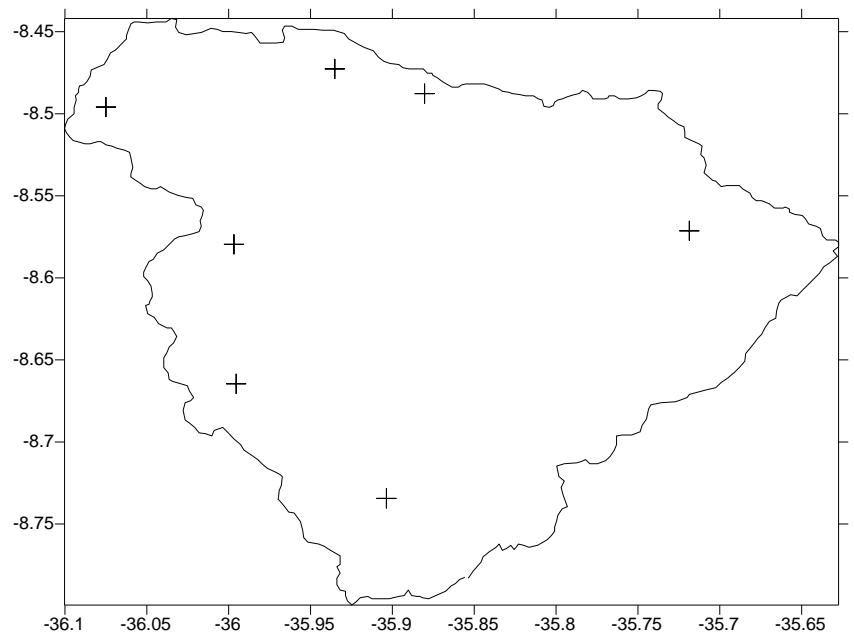

Figura 2: Região do Alto Mundaú e as estações pluviométricas utilizadas neste trabalho. 
Foram utilizados dados de precipitação para o período de 19551991 para 7 localidades da região do Alto Mundaú (Figura 2), situada em Pernambuco, no âmbito da bacia hidrográfica do rio Mundaú, obtidos através da Agência Nacional das Águas (ANA). Deve-se lembrar da má distribuição de postos pluviométricos na região.

De acordo com a justificativa de Da Silva (2009), o IAC pode ser escolhido para acompanhar a variabilidade pluviomérica e não o índice de Palmer (Palmer Drought Severity Index-PDSI), porque este último, por exemplo, é calculado com base em dados de evapotranspiração, infiltração, escoamento superficial eventual etc. e expressa uma medida para a diferença acumulada entre a precipitação normal e a precipitação necessária à evapotranspiração (HAVENS, 1969; STEILA, 1971; ALLEY, 1984 E 1985; GUTTMAN, 1991). Já o IAC só necessita de dados de precipitação, é simples de ser calculado e visa tornar o desvio da precipitação em relação à condição normal de diversas regiões passíveis de comparação.
Considerado mais coerente, foi usado o Rainfall Anomaly Index (RAI) ou Índice de Anomalia de Chuva (IAC) desenvolvido e utilizado por Rooy (1965) e adaptado por Freitas (2004; 2005).

$I A C=3\left[\frac{(\bar{N}-\bar{N})}{\bar{M}-\bar{N}}\right]$, para anomalias

positivas;

$$
I A C=-3\left[\frac{(-\bar{N})}{\overline{(}-\bar{N}}\right\} \text {, para anomalias }
$$

negativas,

Em que: $\mathrm{N}=$ precipitação mensal atual, ou seja, do mês que será gerado o IAC $(\mathrm{mm}) ; \overline{\mathrm{N}}=$ precipitação média mensal da série histórica $(\mathrm{mm}) ; \bar{M}=$ média das dez maiores precipitações mensais da série histórica $(\mathrm{mm}) ; \overline{\mathrm{X}}=$ média das dez menores precipitações mensais da série histórica $(\mathrm{mm})$; e anomalias positivas são valores acima da média e negativas, abaixo da média.

A partir da metodologia de Rooy (1965) e Freitas (2004, 2005) foi calculado o IAC para construir a série de 1955 a 1991, classificando o ano como seco ou chuvoso.

Posteriormente, foram analisados anos classificados como seco e chuvoso calculando o IAC mensal. Pretende-se 
com isso, analisar diferentes anos verificando a contribuição da quadra chuvosa para o total anual e conhecer melhor os mecanismos meteorológicos atuantes no período.

Foram feitas correlações entre os índices climáticos IOS (Índice de Oscilção $\quad$ Sul), IME (Índice Multivariado de El Niño-Oscilação Sul), ATSM (Anomalia de Temperatura de Superfície do Mar - do Oceano Atlântico Tropical) e ODP (Oscilação Decadal do Pacífico) e as anomalias de chuva de todos os anos para o AM através de regressão linear realizadas no Excel ou no software SPSS versão 9.0, pelo método de Stepwise, no intuito de mostrar as relações entre a variabilidade climática global e as chuvas locais.

Foram analisados os valores dos coeficientes de correlação e determinação aplicando o teste de tStudent, no sentido de verificar se o valor do coeficiente de determinação entre as duas variáveis é significativo estatisticamente para a amostra.

Para amostras de tamanho $\mathrm{N}>30$, denominadas grandes amostras, as distribuições amostrais de várias estatísticas são aproximadamente normais e melhores como o crescimento de N. Para amostras de tamanho $\mathrm{N}<30$, denominadas pequenas amostras, essa aproximação não é boa e torna-se pior com o decréscimo de $\mathrm{N}$, de modo que devem ser introduzidas as modificações convenientes.

Uma das mais utilizadas distribuições para pequenas amostras é a "t-Student", a qual é bastante utilizado nos estudos de meteorologia (KOUSKY E KAYANO, 1994; KAYANO E KOUSKY, 1996; FIGUEROA, 1997; CASTRO, 2002), e pode ser calculado da seguinte forma:

$$
t_{c}=\frac{t}{\sqrt{(n-2)+t^{2}}}
$$

Em que: $\mathrm{t}_{\mathrm{c}}=$ valor do percentil e ${ }_{\mathrm{c}}$ é o grau de liberdade. Foi usado $\mathrm{p}=0,95 \mathrm{ou}$ $95 \% ; \mathrm{t}=$ valor do percentil tabelado de acordo com v (n-1); n é o número de dados.

Posteriormente, os dados de precipitação foram sujeitas a $\mathrm{AO}$ para todo o período de dados com a intenção de identificar tendências, ciclos ou oscilações no âmbito da região durante as diferentes configurações atmosféricas de grande escala e para poder proceder comparações. Ao mesmo tempo verificou-se as escalas temporais dominantes. 
$\mathrm{O}$ índice de precipitação submetido à $\mathrm{AO}$ foi primeiramente tratado. Os mesmos são anomalias das variáveis calculadas a partir da climatologia mensal e normalização através dos respectivos desvio padrões, como mostrado abaixo:

$\operatorname{AVar}_{i, j}=\left(\operatorname{Var}_{i, j}-\overline{\operatorname{Var}}\right) / \sigma_{i}$

Em que: $\mathrm{AVar}_{\mathrm{i}, \mathrm{j}}$ é a anomalia da variável, no ano $\mathrm{j}=1,2,3, \ldots, \mathrm{N}$ e mês $\mathrm{i}$ $=1,2,3, \ldots, 12 ; \operatorname{Var}_{i, j}$ é a variável no ano $\mathrm{j}=1,2,3, \ldots, \mathrm{N}$ e mês $\mathrm{i}=1,2,3, \ldots$, 12 , o qual foi calculado sua anomalia; $\overline{\operatorname{Var}_{2}}$ é a média climatológica do mês que calculou-se a anomalia e $\sigma_{i}$ é o desvio padrão utilizado para cada mês i especifico.

Como ocorrido também em Da Silva (2009), será usada a série de anomalias de precipitação do Alto Mundaú como índice de precipitação na aplicação da análise de ondeletas, pelo fato de que a variabilidade de precipitação na região nordeste do Brasil têm sinal homogêneo. Essa metodologia também foi aplicada por Kousky e Chu (1978), Aceituno (1988), Andreoli et al. (2004).

O termo ondeleta refere-se a um conjunto de funções com forma de pequenas ondas geradas por dilatações (a) e translações (b) de uma função simples $\psi_{(\mathrm{t})}$ de variável real t, algumas vezes chamada de ondeleta-mãe. As funções derivadas da ondeleta-mãe são denominadas ondeletas filhas, ou simplesmente ondeletas (WENG e LAU, 1994). Esta função deve ser quadraticamente integrável dentro de um intervalo real, ou espaço $\left[\mathrm{L}^{2}(\Re)\right]$, ou seja, deve apresentar energia finita. Define-se matematicamente a função ondeleta numa escala $a$ e posição b, onde $a$ e $b$ são valores reais, e $a>0$, como sendo:

$\psi_{\mathrm{a}, \mathrm{b}}(\mathrm{t})=\frac{1}{\sqrt{a}} \psi\left(-b / a^{-}\right.$

A transformada em ondeletas contínua da função $(t) f$ é definida pela seguinte equação:

$(\mathrm{W} \psi f)(\mathrm{a}, \mathrm{b})=\frac{1}{\sqrt{a}} \int f(t) \psi-b / a-d t$

Em que: $f(t)$ é a função que constitui a série de dados a ser analisada. $\mathrm{O}$ termo $1 / \sqrt{a}$ é utilizado para normalizar a energia de cada ondeleta.

A ondeleta Morlet é complexa e possui características semelhantes às de sinais meteorológicos, tais como simetria ou assimetria, e variação 
temporal brusca ou suave. Segundo a literatura, este é um critério para escolha da função ondeleta (WENG E LAU, 1994; MORETTIN, 1999). A função de Morlet é dada pela seguinte expressão:

$\psi(\mathrm{t})=\mathrm{e}^{\mathrm{iwot}} \mathrm{e}^{-t^{2} / 2}$

que representa uma onda modulada por um envelope Gaussiano.

Pode-se então variar a "escala" da ondeleta mudando sua largura. Esta é a vantagem real da $\mathrm{AO}$. Ondas Morlet apresentam alta freqüência enquanto gaussianas apresentam baixa freqüência. Para a determinação de ciclos curtos ou longos modifica-se o tipo da onda para Morlet ou Gaussiana, respectivamente.

A maneira de mudar o tamanho total das ondeletas em relação ao tempo, as chamadas "as ondeletas escaladas" é representada como:

$\psi\left[\frac{\left(n^{\prime}-n\right) \delta t}{s}\right]=\left(\frac{\delta t}{s}\right)^{1 / 2} \psi_{0}\left[\frac{\left(n^{\prime}-n\right) \delta t}{s}\right]$

Em que s é o parâmetro de "dilatação" usado para mudar a escala, e $\mathbf{n}$ é o parâmetro de transformação no tempo. $\mathrm{O}$ fator de $\mathbf{s}^{-1 / 2}$ é uma normalização para manter a energia total da Wavelet. A transformação do conjunto da onda é dada por:
$W_{n}(s)=\sum_{n^{\prime}=0}^{N-1} x_{n^{\prime}} \psi^{*}\left[\frac{\left(n^{\prime}-n\right) \delta t}{s}\right]$

(9)

Em que o asterisco (*) denota o complexo conjugado de phi. A integral acima pode ser utilizada para valores de escalas (desde alta frequiência até a mais baixa). Um retrato bidimensional da variabilidade pode então ser construído traçando a amplitude e a fase da Wavelet.

\section{RESULTADOS E DISCUSSÃO}

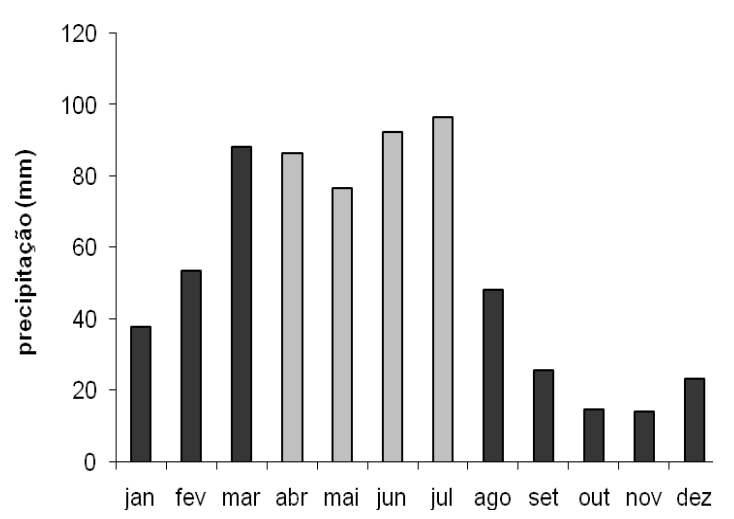

Figura 3: Médias climatológicas da precipitação no Alto Mundaú. 


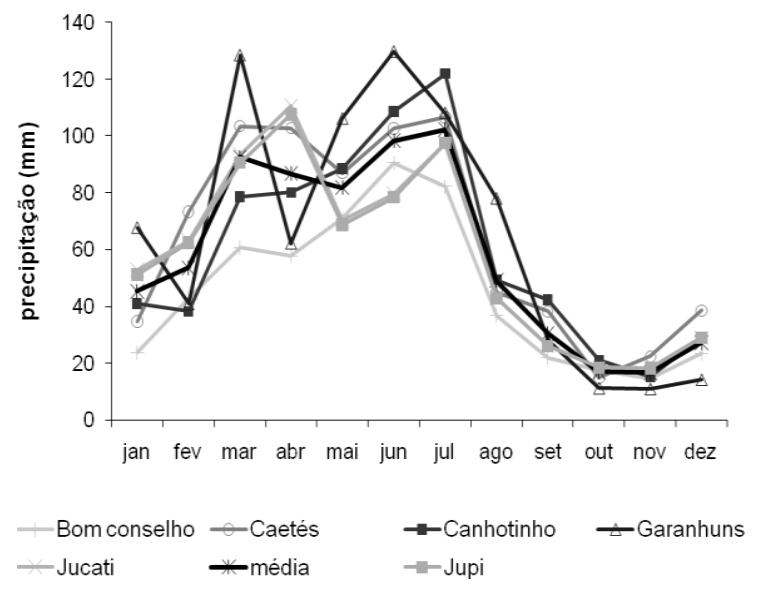

Figura 4: Médias climatológicas municípios que compõem o ASF.

Na Figura 3 pode-se visualizar a variabilidade anual da precipitação no AM, com quadra mais chuvosa entre abril a julho (cor clara na Figura 3), tendo o mês de março uma forte contribuição nos totais anuais. Já no quadrimestre setembro-dezembro são registrados os menores valores mensais de chuva.

A Figura 4 mostra as médias climatológicas dos municípios do AM que foram utilizados neste estudo. As médias climatológicas apresentaram maior variabilidade entre os seus municípios, coincidindo aumento de precipitação em maio, junho e julho. As localidades de Garanhuns e Caetés apresentaram valores climatológicos maiores e Bom Conselho, as menores médias climatológicas.
A Figura 5 mostra o IAC do AM de 1955 a 1991. Um aumento de ocorrência de anos úmidos depois de 1974 foi observado no AM, quando antes de 1974, ocorreram 13 anos secos e 6 anos úmidos, e após 1974, houveram 9 anos secos e 9 anos úmidos; ou seja, diminuíram o número de anos secos. Este comportamento leva dos ao ano de 1974 ser considerado um "ponto de inversão" no padrão de precipitação.

Este "ponto de inversão" também foi observado na vazão do rio Mundaú por Da Silva et al. (2005), e na precipitação da bacia hidrográfica do rio Paraíba por Araújo et al. (2007).

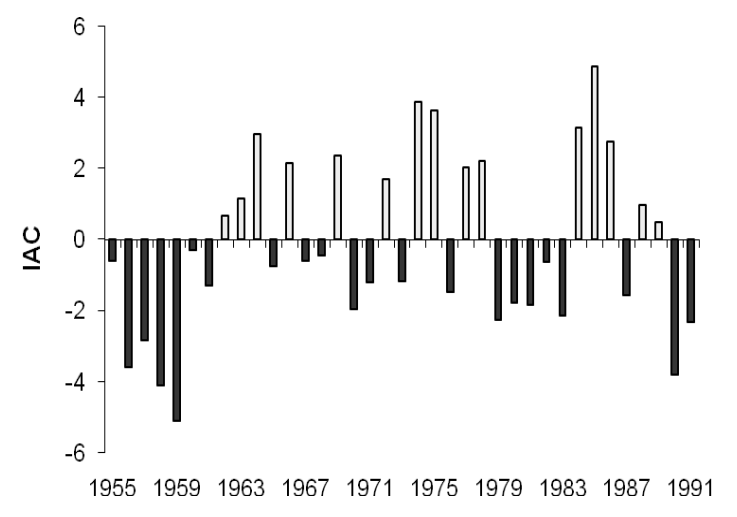

Figura 5: IAC anual para o Alto Mundaú

Dentre os anos da série de IAC (Figura 5), foram escolhidos dois anos secos (1979 e 1990) e dois anos úmidos (1975 e 1977) para analisar o 
comportamento desses anos e as diferenças entre suas quadras chuvosas.

Durante os anos secos de 1979 e 1990 (Figura 6), somente os meses de janeiro, abril e maio de 1979 apresentaram IAC positivo. Esse resultado reforça o argumento de Aragão (1975), no qual é afirmado que, durante os períodos de seca no NEB há suficiente umidade nos baixos níveis da atmosfera, mas inexiste um mecanismo dinâmico capaz de provocar movimentos ascendentes que tenham como resultado formação de nuvens suficientementes desenvolvidas para produzir precipitação. Deste modo, o IAC, em anos secos, tende a ser negativo, até mesmo na quadra chuvosa, não sendo, necessariamente, em anos úmidos. Em ambos anos secos, o total anual atinge a metade do valor dos anos úmidos (1975 e 1985), com 334 mm em 1979 e 252 mm em 1990. A quadra chuvosa contribui com aproximadamente $68 \%$ do valor anual em ambos os casos.

Os IACs mensais de 1975 e 1985 (Figura 7), anos úmidos, apresentaramse negativos na quadra mais seca, em ambos os anos. Já na quadra chuvosa (abril a julho), foram atingidos os máximos valores, com exceção de maio de 1985, que foi negativo e em junho e julho de 1985, quando o IAC apresentou uma queda nos valores se comparado a 1975.

A quadra chuvosa de 1975 contribuiu para o total anual com 739 mm de chuva, o que corresponde a $75 \%$ do valor médio anual. Já 1985, quando se verificou uma notável diminuição desses valores, foram registrados 562 mm de chuva, o que totaliza $51 \%$ do total médio anual.

Os principais mecanismos que propiciam a ocorrência de chuvas são as brisas que levam bandas de nebulosidade até a parte alta da bacia, a ação de frentes frias ou seus remanescentes, Ondas de Leste, que atuam durante o inverno, e das Perturbações Ondulatórias dos Alísios (POAS), que atuam principalmente de junho a agosto.

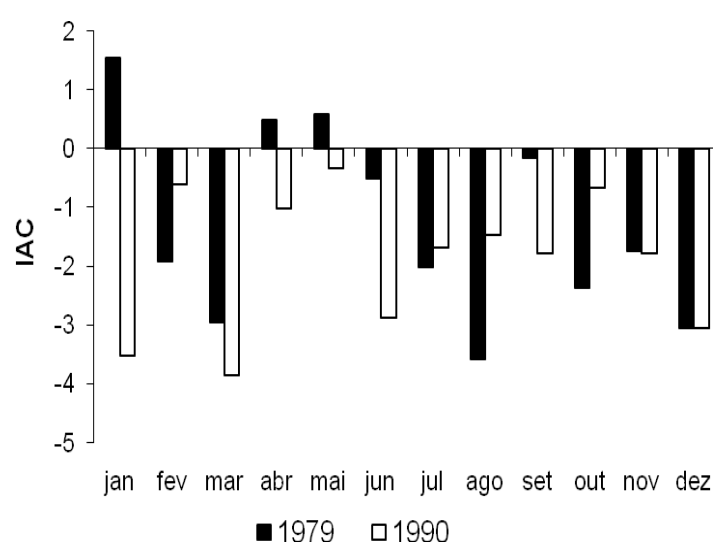

Figura 6: IAC mensal de anos secos (conforme IAC anual) para o Alto Mundaú. 


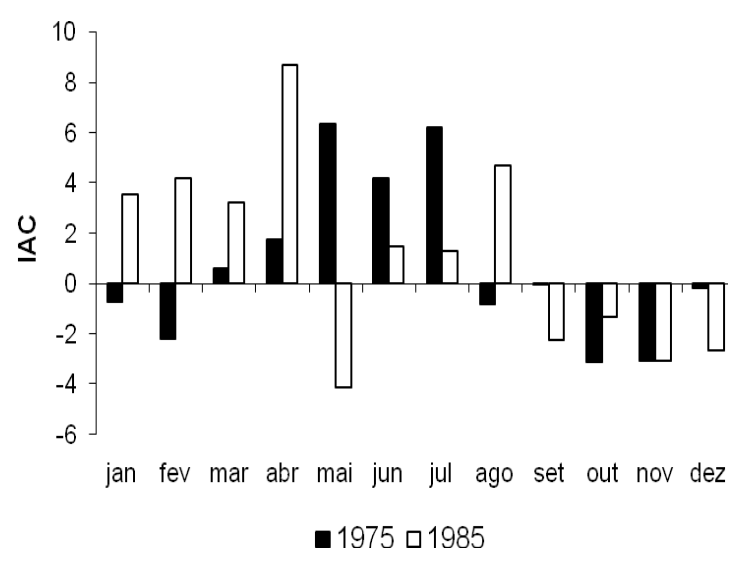

Figura 7: IAC mensal de anos úmidos (conforme IAC anual) para o Alto Mundaú.

Foram feitas regressões lineares entre os valores reais de anomalias de precipitação no AM e índices climáticos.

Tanto para os anos secos, quanto para os anos úmidos, os valores dos coeficientes de correlação e determinação foram os melhores utilizando o índice de ODP, o qual pode explicar entre $89 \%$ e $85 \%$ (Tabela 1) das chuvas no Alto Mundaú, respectivamente.

Aplicando o teste de significância t-Student, o mínimo considerável de coeficiente de correlação para a amostra seria 0,612 , quando foi apresentado 0,79 e 0,73 , respectivamente, tornando a correlação significativa estatisticamente. Na Tabela 1, são mostrados valores de coeficiente de determinação e correlação entre anomalias de precipitação do AM e índices climáticos. Na mesma observouse a forte influência da Oscilação Decadal do Pacífico sobre os totais pluviométricos do AM.

Tabela 1: Valor do coeficiente de determinação, correlação e percentual de determinação $\left(\mathrm{r}^{2}\right)$ entre anomalias de precipitação no AM e quatro índices climáticos.

\begin{tabular}{|c|c|c|c|c|}
\hline Índices & Ano & $\begin{array}{l}\text { Coeficiente de } \\
\text { correlação }\end{array}$ & $\begin{array}{l}\begin{array}{l}\text { Coeficiente de } \\
\text { determinação }\end{array} \\
\end{array}$ & $\mathbf{r}^{2}(\mathrm{em} \%)$ \\
\hline \multirow[t]{2}{*}{ IME } & seco & $-0,26$ & 0,509 & 50,9 \\
\hline & úmido & $-0,5$ & 0,7 & 70 \\
\hline \multirow[t]{2}{*}{ IOS } & seco & 0,692 & 0,831 & 83,1 \\
\hline & úmido & 0,29 & 0,538 & 53,8 \\
\hline \multirow[t]{2}{*}{$O D P$} & seco & $0,791 *$ & 0,889 & 88,9 \\
\hline & úmido & $0,732 *$ & 0,855 & 85,5 \\
\hline \multirow[t]{2}{*}{ TSM } & seco & 0,166 & 0,407 & 40,7 \\
\hline & úmido & 0,486 & 0,697 & 69,7 \\
\hline
\end{tabular}

* Melhor valor significativo de acordo com o teste de t-Student. 
$\mathrm{Na}$ análise de ondeletas encontrou-se o pico dominante na EPG na escala temporal de 11 anos. O EPG (Figura 8c) mostrou também um pico dominante na escala de 22 anos. De acordo com a literatura, citados em Da Silva (2003), períodos de aproximadamente 21 anos, podem estar relacionados com ciclos de atividade solar, variações de atração gravitacional entre Terra-Lua-Sol ou variações internas do sistema Terra-oceanoatmosfera. Já os de 11 anos são associados ao ciclo de manchas solares (KERR, 1996 e MOLION, 2005).

a) Índice de Prp do AM

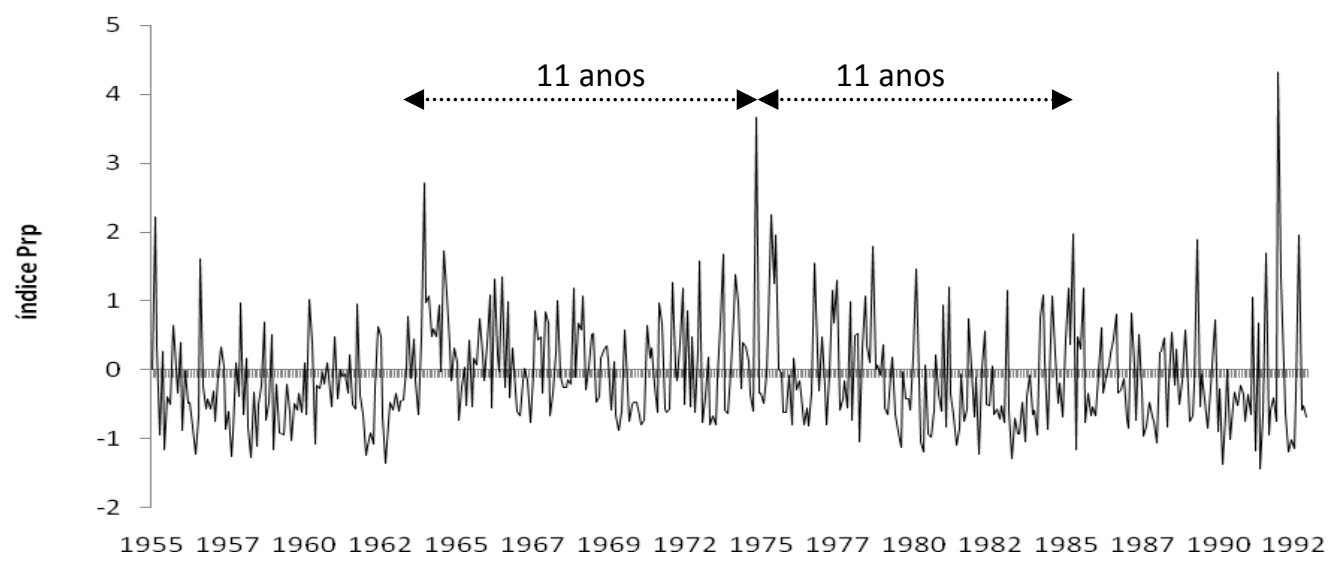

b) Espectro de energia (EPW)

c) Espectro global de wavelet (EPG)
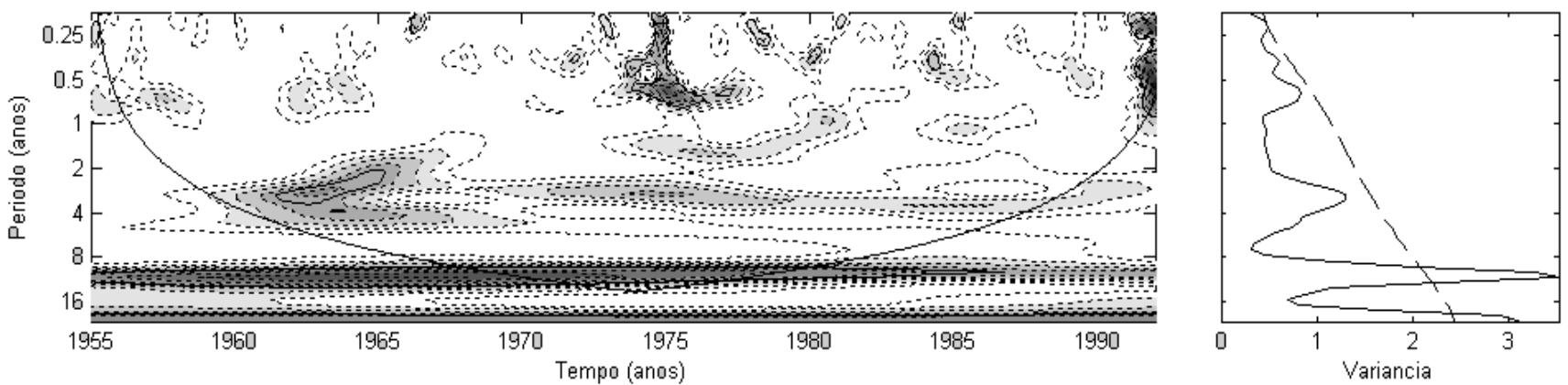

Figura 8: a) Índice de precipitação para AM (Prp) normalizado pelo desvio padrão; b) Espectro de energia local para Prp. Contornos sombreados correspondem a valores de variância normalizados. Contornos tracejados correspondem a variâncias significativas ao nível de $95 \%$. A curva em forma de $\mathrm{U}$, representado o cone de influência, sob o qual o efeito de borda é importante; c) Espectro global de wavelet, o contorno tracejado indica que o espectro de wavelet global é significativo ao nível de confiança de $95 \%$. 
Os picos estatisticamente não significativos ocorrem principalmente para as escalas de tempo de 5 anos e de aproximadamente 2 anos. Os picos de 11 e 22 anos são resultantes de altos valores de EPW (Figura 8b) na maior parte do período de estudo. Já os eventos de escala de tempo interanual decorreram de altos valores de EPW em 1960-1965, 1973-1990 para a escala de 5 anos e em 1955-1958, 1962-1965, 1972-1975, 1990-1992 para as escalas mais curtas. É importante notar que as variações de escalas mais curtas podem ser importantes na pluviometria, contribuindo para os altos valores de Prp (Figura 8a). Esses resultados são semelhantes aos encontrados por Andreoli et al. (2004) para Fortaleza.

As variações em escalas média e interanual contribuem positivamente para as precipitações locais. Estes resultados indicam que a variabilidade de precipitação na bacia é definida por multi-escalas temporais localizadas em certos intervalos de tempo; no entanto, a variância significativa dominante ocorre na escalas decadal.

Verificou-se que principalmente a variabilidade interanual ligada ao ciclo de ENOS e a variabilidade decadal influenciam na variabilidade pluviométrica local. Esses resultados encontram-se de acordo com os observados por Andreoli et al. (2004), Markham (1974), Chu (1984) e Hastenrath e Kaczmarczyk (1981), todos realizados para Fortaleza, também no NEB.

Diante do fato de que eventos de escala temporal de 11 e 22 anos no AM pode-se planejar o armazenamento e aproveitamento das águas de chuva, além de prevenir a população ribeirinha e agricultores para eventos extremos e enchentes no AM. Essas escalas temporais que dominam sobre escalas de eventos meteorológicos atuantes são contribuintes para aumento do total pluviométrico e, através do acompanhamento pluviométrico, podese máximar o aproveitamento de água de chuva na gestão agrícola, pesqueira, social e energética, dentre outras.

\section{CONCLUSÕES}

No Alto Mundaú o período depois de 1974 foi mais úmido que o período anterior a este ano. Isto tem relação com a junção de ações antrópicas e naturais.

O IAC, em anos secos, tende a ser negativo, até mesmo na quadra chuvosa, ou seja, em anos secos, as chuvas são 
abaixo da média até mesmo durante a sua quadra chuvosa.

Diante das correlações lineares, concluiu-se que a ODP interfere nas chuvas da região, tanto em anos secos quanto úmidos.

A partir das ondeletas obtiveramse resultados que indicaram que a variabilidade de precipitação na região é definida por multi-escalas temporais localizadas em certos intervalos de tempo. Principalmente a variabilidade interanual ligada ao ciclo de ENOS e a variabilidade decadal das escalas $11 \mathrm{e}$ 22 anos influenciaram na variabilidade pluviométrica local.

Concluiu-se que as ferramentas estatísticas são de extrema utilidade para o conhecimento da climatologia e mostram quais os possíveis eventos da variabilidade climática que exerce maior influência sobre a pluviometria local.

\section{REFERÊNCIAS BIBLOGRÁFICAS}

ACEITUNO, P. On the functioning of the Southern Oscillation in the South American Sector. Part 1: surface climate, Mon. Wea. Rev., v.116, p.505524, 1988.

ALLEN, M.R.; SMITH, L.A. Investigating the origins and significance of low frequency modes of climate variability. Geophysical
Research Letters, Washington, v.21, n.10, p.883-886, 1994.

ALLEY, W.M. The Palmer Drought Severity Index: Limitations and Assumptions, Journal of Climate and Applied Meteorology, 23, 1100-1109, 1984.

ALLEY, W.M. The Palmer Drought Severity Index as a Measure of Hidrologic Drought, Water Resources Bulletin, 21 (1), 105-114, 1985.

ANDREOLI, R. V., KAYANO, M. T., GUEDES, R. L., OYAMA, M. D., ALVES, M. A. S. A influência da temperatura da superfície do mar dos Oceanos Pacífico e Atlântico na variabilidade de precipitação em Fortaleza, Revista Brasileira de Meteorologia, v.19, n.3, 337-344, 2004.

ARAGÃO, J.O.R. Um estudo da estrutura das perturbações sinóticas do Nordeste do Brasil. Dissertação de Mestrado em Meteorologia - São José dos Campos - SP, INPE, 47 p., 1975.

ARAÚJO, L. E.; DA SILVA, D. F.; MORAES NETO, J. M.; SOUSA, F. A. S. de. Análise da Variabilidade EspaçoTemporal da precipitação na Bacia do rio Paraíba usando IAC. Revista de Geografia, UFPE, Recife-PE, vol.1, 2007.

BARBOSA, E.B.M.; ROSA, M.M.; VIJAYKUMAR, N.L.; BOLZAN, M.J.A.; TOMASELLA, J. Caracterização por Ondeletas de Processos Físicos Não-Lineares na Micro-bacia Amazônica, INPE, São José dos Campos, 2004. 
CASTRO, C.A.C. 2002. Interações Trópicos-Extratrópicos na escala de tempo intra-sazonal durante o verão austral e seus efeitos na América do Sul. Dissertação de Mestrado. Instituto Nacional de Pesquisas Espaciais, São Paulo.

CHU, P. S. Time and space variability of rainfall and surface circulation in the northeast Brazil - Tropical Atlantic sector, J. Meteor. Soc. Japan, v.26, n.2, p.363-369, 1984.

COSTA, M. C.; OLIVEIRA, M. C. F.; MORAES, J. C.; BARRETO, P. N.; DANTAS, V. A.; CARVALHO, S. P. Comportamento e relação entre PRP e vazão na bacia do rio Mundaú, Alagoas e Pernambuco. XIV Congresso de Brasileiro de Agrometeorologia, Anais..., Campinas-SP, julho 2005.

DA SILVA, D.F. Influência da Variabilidade Climática Interanual na Hidrologia da Bacia do rio São Francisco, Trabalho de Conclusão de Curso (Bacharelado em Meteorologia), Universidade Federal de Alagoas UFAL, 2003.

DA SILVA, D.F.; BORBA, J.C.C.; ALMEIDA, H.R.R.C.; LOPES, Z.F. Influência do Clima na Variabilidade Espaço-Temporal da bacia do rio Mundaú (localidades de Pernambuco). I Simpósio Internacional de Climatologia, Anais..., Fortaleza-Brasil, Outubro de 2005.

DA SILVA, D.F. Análise de aspectos climatológicos, ambientais, agroeconômicos e de seus efeitos sobre a Bacia hidrográfica do rio Mundaú (AL e PE). Tese de
Doutorado em Recursos Naturais, março 2009, UFCG (PB).

FARGE, M.: Wavelet transforms and their applications to turbulence. Ann.Rev.Fluid Mech., 24, 395-457, 1992.

FIGUEROA, S.N. Estudo dos sistemas de circulação de verão sobre a América do Sul e suas simulações com modelos numéricos. Tese de Doutorado do Instituto Nacional de Pesquisas Espaciais (INPE-7121TDI/672). São José dos Campos, 1997.

FREITAS, M. A. S. A Previsão de Secas e a Gestão Hidroenergética: $\mathrm{O}$ Caso da Bacia do Rio Parnaíba no Nordeste do Brasil. In: Seminário Internacional sobre Represas y Operación de Embalses, 2004, Puerto Iguazú. Anais do Seminário Internacional sobre Represas y Operación de Embalses. Puerto Iguazú : CACIER, 2004. v. 1. p. 1-1.

FREITAS, M. A. S. Um Sistema de Suporte à Decisão para o Monitoramento de Secas Meteorológicas em Regiões SemiÁridas. Revista Tecnologia, Fortaleza, v. Suplem, p. 84-95, 2005.

GONÇALVES, W.A.; CORREIA, M.F.; ARAÚJO, L.E.; DA SILVA, D.F.; ARAÚJO, H.A. Vulnerabilidade Climática do Nordeste Brasileiro: Uma análise de eventos extremos na Zona Semi-árida da bacia hidrográfica do São Francisco, XIV Congresso Brasileiro de Meteorologia, Anais...., Florianopólis, 2006. 
GUTTMAN, N.B. A Sensitivity Analysis of the Palmer Hydrologic Drought Index, Water Resources Bulletin, 27(5), 797-807, 1991.

KANE, R. P.; TRIVEDI, N. B. Spectral characteristics of the annual rainfall series for northeast Brazil, Clim. Change, v.13, p.317-336, 1988.

KANE, R. P. El Niño and La Niña events and rainfall in NE and South Brazil, Rev. Bras. Geofís., v.10, n.2, p.49-59, 1992.

KANE, R. P. Prediction of droughts in North-east Brazil: Role of ENSO and use of periodicities, Inter. J. Climatol., v.17, p.655-665, 1997.

KAYANO, M. T.; KOUSKY, V. E. Tropical circulation variability with emphasis on interannual and intraseasonal time scales. Revista Brasileira de Meteorologia, v. 11, n. 1 e 2, p. 06 -17, Jun. 1996.

KERR, R.A. A now dawn for sunclimate links? Science, Washington, DC, v.271, n5254, p.1360-1361, 1996.

KOUSKY, V.E.; CHU, P.S. Fluctuations in annual rainfall for northeast Brazil, J. Meteor. Soc. Japan, v.56, p.457-466, 1978.

KOUSKY, V. E.; KAYANO, M. T. Principal modes of outgoing longwave radiation and $250-\mathrm{mb}$ circulation for the South American sector. J. Climate, 7, 1131-1143, 1994.
HASTENRATH, S., KACZMARCZYK, E. B. On spectra and coherence of tropical climate anomalies, Tellus, v.33, n.5, p.453-462, 1981.

HAVENS, A.V. Economic Impact of Drought on Water Systems in Passaic River Basin, n.J., New Brunswick, New Jersey Agricultural Experiment Station, 1969.

MANN, M.E.; LALL, U.; SALTZMANN, B. Decadal-tocentennial-scale climate variability: insights into the rise and fall of the great salt lake. Geophysical Research Letters, Washington, DC, v.22, n.8, p.937-940, apr.1995.

MARKHAM, C. G. Apparent periodicities in rainfall at Fortaleza, Ceará, Brazil, J. App. Meteor., v.13, p.176-179, 1974.

MAUGET, S.U.M. Índice Padronizado de Precipitação (SPI); C. Springer Science, 2005.

MEHTA,V.; DELWORTH, T. Decadal variability of the Tropical Atlantic ocean surface temperature in shipboard measurements and in a global oceanatmosphere model, J. Clim., v.8, n.3, p.172-190, 1995.

MOLION, L.C.B. Aquecimento Global, El Niños, Manchas Solares, Vulcões e Oscilação Decadal do Pacífico, Revista Climanálise, 2005. 
MORETTIN, P.A. Ondas e Ondeletas: Da Análise de Fourier à Análise de Ondeletas, EDUSP, 1999.

REPELLI, C. A.; FERREIRA, N. S.; ALVES, J. M. B.; NOBRE, Carlos Afonso. Índice de anomalia de precipitação para o Estado do Ceará. In: $X$ CONGRESSO BRASILEIRO DE METEOROLOGIA E VIII CONGRESSO DA FLISMET, 1998, Brasília DF. Anais do $\mathbf{X}$ Congresso Brasileiro de Meteorologia e VIII Congresso da FLISMET, 1998.

ROOY, M.P. VAN. A Rainfall Anomaly Index Independent of Time and Space, Notes, 14, 43, 1965.

SCHNEIDER M., VITORINO, I., SILVA DIAS, P.L. Monitoramento da Intrasazonalidade por meio da Transformada em Ondeletas, Simpósio Internacional de Climatologia, Anais..., Fortaleza-CE, out.2005.

SPERBER, K. R.; HAMEED, S. Phase Locking of Nordeste precipitation with sea surface temperatures, Geophys. Res. Lett., v.20, n.2, p.113-116, 1993.

STEILA, D., 1971: Drought Analysis in Four Southern States by a New Index, PhD Diss., Athens, Ga., The University of Georgia.

VITORINO, M.I. Análise das Oscilações Intrasazonais sobre a América do Sul e Oceanos Adjacentes Utilizando a Análise de Ondeletas, Tese de Doutorado em Meteorologia, INPE, São José dos Campos, 2003.
TENÓRIO, R. S. Estudo Hidrometeorológico da Bacia do Rio Mundaú. Universidade Federal de Alagoas. MET. Maceió - Alagoas, Fevereiro/1985.

TUCCI, C.E.M; BRAGA, B. Clima e Recursos Hídricos no Brasil, Coleção ABRH, p. 348, 2003.

WENG, H. LAU, K-M. Wavelets, period doubling, and time-frequency localization with application to organization of convection over the Tropical Western Pacific. Journal of the Atmospheric Sciences, v.51, n.17, p.2523-2541, 1994.

ZHANG, Y.; WALLACE, J.M.; BATTISTI, D. ENSO-like interdecadal variability: 1900-93, J. Clim., v.10, p.1004-1020, 1997. 